ARTICLE

https://doi.org/10.1038/s41467-020-19690-0

\title{
A Cas-embedding strategy for minimizing off- target effects of DNA base editors
}

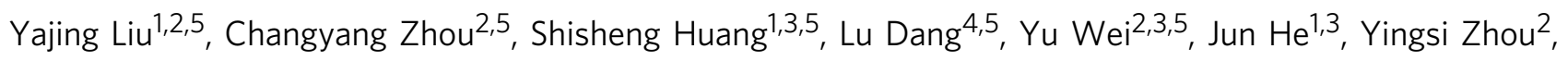

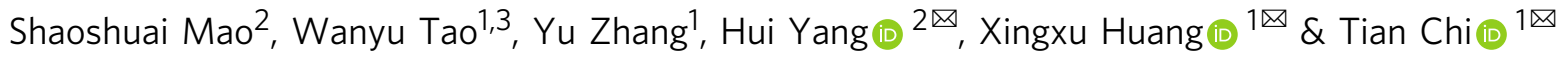

DNA base editors, typically comprising editing enzymes fused to the $\mathrm{N}$-terminus of nCas9, display off-target effects on DNA and/or RNA, which have remained an obstacle to their clinical applications. Off-target edits are typically countered via rationally designed point mutations, but the approach is tedious and not always effective. Here, we report that the offtarget effects of both $A>G$ and $C>T$ editors can be dramatically reduced without compromising the on-target editing simply by inserting the editing enzymes into the middle of nCas9 at tolerant sites identified using a transposon-based genetic screen. Furthermore, employing this Cas-embedding strategy, we have created a highly specific editor capable of efficient $C>T$ editing at methylated and GC-rich sequences.

\footnotetext{
${ }^{1}$ School of Life Science and Technology, ShanghaiTech University, Shanghai, China. ${ }^{2}$ Institute of Neuroscience, State Key Laboratory of Neuroscience, Key Laboratory of Primate Neurobiology, CAS Center for Excellence in Brain Science and Intelligence Technology, Shanghai Research Center for Brain Science and Brain-Inspired Intelligence, Shanghai Institutes for Biological Sciences, Chinese Academy of Sciences, Shanghai, China. ${ }^{3}$ University of Chinese Academy of Sciences, Beijing, China. ${ }^{4}$ Cancer Hospital and Institute of Guangzhou Medical University, Guangzhou, China. ${ }^{5}$ These authors contributed equally: Yajing Liu,

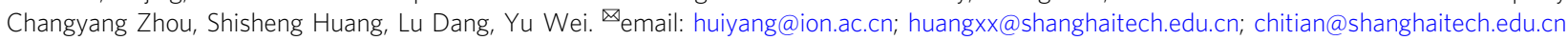


D NA base editors achieve targeted nucleotide substitutions without introducing double-strand DNA breaks, thus holding great potential for correcting point mutations underlying many human genetic diseases ${ }^{1,2}$. However, both adenine base editor $(\mathrm{ABE})$ and cytosine base editor (CBE) can create substantial off-target edits on $\mathrm{RNA}^{3,4}$, whereas $\mathrm{CBE}$ additionally has off-target effects on DNA ${ }^{1,3-8}$. Such effects raise safety concerns regarding the clinical use of the base editors, which have motivated intense searches for countermeasures ${ }^{1,9}$. Off-target editing results from the intrinsic properties of the editing enzymes harnessed for base editing, and consequently, have been countered by mutating these enzymes. Multiple highspecificity editors bearing rationally engineered point mutations as well as deletions at the deaminase moiety have been reported, including $\mathrm{YE} 1$ and $\mathrm{ABE}^{\mathrm{F} 148 \mathrm{~A} 3,4,10-14}$. However, this conventional strategy for minimizing the off-target effects requires prior knowledge about the enzyme structure, is labor intensive, and not always effective. Alternative approaches that are straightforward and generally applicable would be highly desirable.

It has been observed that some editing enzymes (APOBEC1 and Tad-TadA*) produce more off-target edits on RNA in HEK293T cells when expressed as free proteins than as $\mathrm{N}$ terminal fusion protein to $\mathrm{nCas}^{4}$, suggesting that fusing the enzymes to nCas9 may hinder off-target editing perhaps due to steric hindrance. We hypothesized that inserting the enzymes into the middle of nCas9, rather than fusing it to its $\mathrm{N}$ terminus, might further reduce the off-target effects, thus offering an attractive alternative to the mutagenesis approach. To test this idea, we first designed a genetic screen to systematically identify the nCas 9 sites tolerant of adenine deaminase insertion, and subsequently demonstrated that the off-target editing of both $\mathrm{ABE}$ and $\mathrm{CBE}$ can be dramatically repressed simply by relocating the deaminase moiety in the base editors from the $\mathrm{N}$ terminus of nCas9 to the tolerant sites in the middle of nCas9, as described below.

\section{Results}

Genetic screen for Cas9 sites tolerant of TadA-TadA* insertion. We sought to use MuA-transposon-based genetic screen ${ }^{15,16}$ to identify the nCas9 sites tolerant of deaminase insertions. First, we constructed an "all-in-one" plasmid expressing: (1) nCas9 under the control of isopropyl $\beta$-D-1-thiogalactopyranoside (IPTG)-inducible promoter, (2) the ampicillinresistant $(\mathrm{AmpR})$ gene bearing a $\mathrm{C}>\mathrm{T}$ mutation that created a premature stop codon (A118X), and (3) a single-guide RNA (sgRNA) under the control of the $\mathrm{J} 23119$ promoter for repairing the premature stop codon. The bacteria transformed with this plasmid would be ampicillin sensitive until $A>G$ editing occurs on the bottom strand of DNA, which corrects the $\mathrm{C}>\mathrm{T}$ mutation in the top strand to restore the translation (Fig. 1a and Supplementary Fig. 1a). We next used Mu transposon to randomly insert the DNA encoding TadA-TadA* into the "all-in-one" plasmid. This insertion plasmid library, prepared in vitro, was then electroporated into Escherichia coli and the cells were grown overnight on plates containing kanamycin but no ampicillin. We evaluated the insertion efficiencies at various positions on nCas9 by deep-sequencing the unscreened plasmid library extracted from the recovered cells. At the nCas 9 coding sequence, we found 51,393 insertions, with at least one insertion in 99\% of amino acid positions (Fig. 1b and Supplementary Table 2), demonstrating that the $\mathrm{Mu}$-mediated mutagenesis was efficient and unbiased. As expected, insertions were hardly detectable in the Kan resistance gene and the replication-related f1 region (Fig. 1b). IPTG was added to the mixture to induce the fusion protein expression (nCas9-TadA-TadA*) before the cells were transferred to plates with ampicillin to select the cells expressing the repaired AmpR gene. Positive clones were picked, and the plasmids were extracted and sequenced to determine the editing efficiencies and TadA-TadA* insertion sites (Fig. 1c).

In total, 43 insertional sites were found on nCas9 by analyzing the plasmids extracted from the recovered ampicillin-resistant colonies. Most of the central fusion ABE variants achieve robust A-to-G editing at the premature stop codon (Fig. 1c). The ABE variants with TadA-TadA* inserted into these highly tolerant sites are identical to ABEmax ${ }^{17}$, except for the location of TadA-TadA*, and were termed CEABEs ("CE" stands for Cas embedding). Among the 43 insertion sites recovered in the screened library, nine sites were clustered together within a short (16-amino acid (a.a.)) segment, occurring at $1048 \mathrm{Thr}, 1050 \mathrm{Ile}, 1051 \mathrm{Thr}, 1052 \mathrm{Leu}$, 1054Asn, 1056Glu, 1057lle, 1059Lys, and 1063Ile. The enrichment of these sites in the screened library was specific, because in the unselected library, these sites were inserted only 61,39 , $90,38,5,29,76,53$, and 25 times, respectively, much less frequently than some other sites (e.g., 1090Pro, inserted 280 times, Supplementary Table 2) that were not recovered after screening. Thus, the 16-a.a. fragment was highly tolerant of insertion and presumably dispensable for nCas9 function. Indeed, this fragment, harbored inside the RuvC III domain in the NUC lobe ${ }^{18}$, is not conserved among 28 SpCas9 orthologs (Supplementary Fig. 1b). Therefore, we replaced the 1048Thr1063Ile region with TadA-TadA* to generate CE-ABE ${ }^{1048-1063}$.

Performance of CE-ABEs in HEK293T cells. We next tested the 20 most frequently recovered CE-ABEs in HEK293T cells. At an endogenous site containing multiple As within the editing window (Site1), 12 of the 20 CE-ABEs were as active as ABEmax, with editing efficiencies ranging from $66-89 \%$ as compared with 86\% for ABEmax (Fig. 2a). The editing efficiencies of the various CE-ABEs in HEK293T cells were largely consistent with their recovery rates in prokaryotic cells, demonstrating the robustness of the screen. We then examined the off-target RNA editing for the 12 variants at three RNA off-target sites known to be highly susceptible to ABEmax. All 12 CE-ABEs showed remarkable reductions in the editing on at least two of the three sites, and four of the 12 variants at all three sites (Fig. 2b, the four variants marked in red). We further used RNA-sequencing (RNA-seq) to profile the off-target edits by these top $4 \mathrm{CE}$-ABEs at the entire transcriptome, and compared them with ABEmax and ABE7.10 $10^{\mathrm{F} 148 \mathrm{~A}}$. ABEmax induced massive off-target edits as reported before, which was reduced at least $6 \times$ in CE-ABEs, with $\mathrm{CE}^{1072}-\mathrm{ABE}$ as much as $236 \times$ (single-nucleotide variant (SNVs) reduced from 20,739 to 88 , a level similar to that produced by ABE7.10 ${ }^{\mathrm{F} 148 \mathrm{~A}}$; Fig. 2c). In contrast, on-target editing by ABEmax was either comparable $(92 \%$ and $89 \%$, for ABEmax and $\mathrm{CE}^{1048-1063}$ - $\mathrm{ABE}$, respectively) or only mildly reduced (to $75 \%$ and $73 \%$ for $\mathrm{CE}^{776}-\mathrm{ABE}$ or $\mathrm{CE}^{1263}-\mathrm{ABE}$, respectively), except that for $\mathrm{CE}^{1072}-\mathrm{ABE}$, the activity was reduced markedly (to $33 \%$ ) (Fig. 2d).

The data above established $\mathrm{CE}^{1048-1063}-\mathrm{ABE}$ as the optimal CE-ABE with balanced efficiency and specificity. Therefore, we characterized its performance further, at 8 and 12 randomly selected genomic sites in HEK293T cells (Fig. 2e, f) and mouse N2a cells (Fig. 2g, h), respectively. We found that $\mathrm{CE}^{1048-1063}$ $\mathrm{ABE}$ was comparable to ABEmax in terms of editing rates (Fig. 2e, g, summarized in Fig. 2i), while the editing window was slightly enlarged (Fig. 2f, h). We concluded that embedding an adenine deaminase in nCas9 could markedly reduce the offtarget effects on RNA with only a minimal impact on on-target editing. 
a
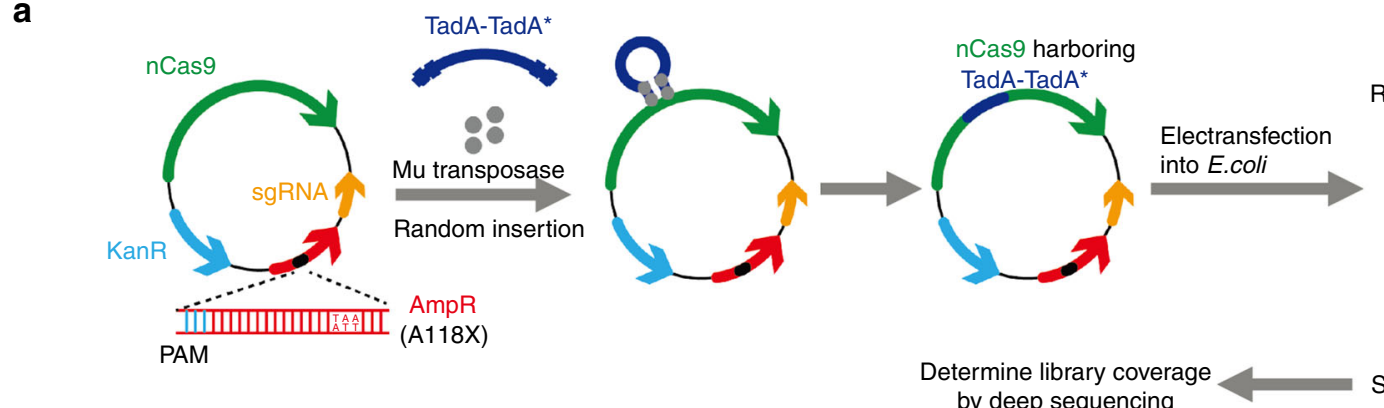

Random insertional library

Determine library coverage by deep sequencing
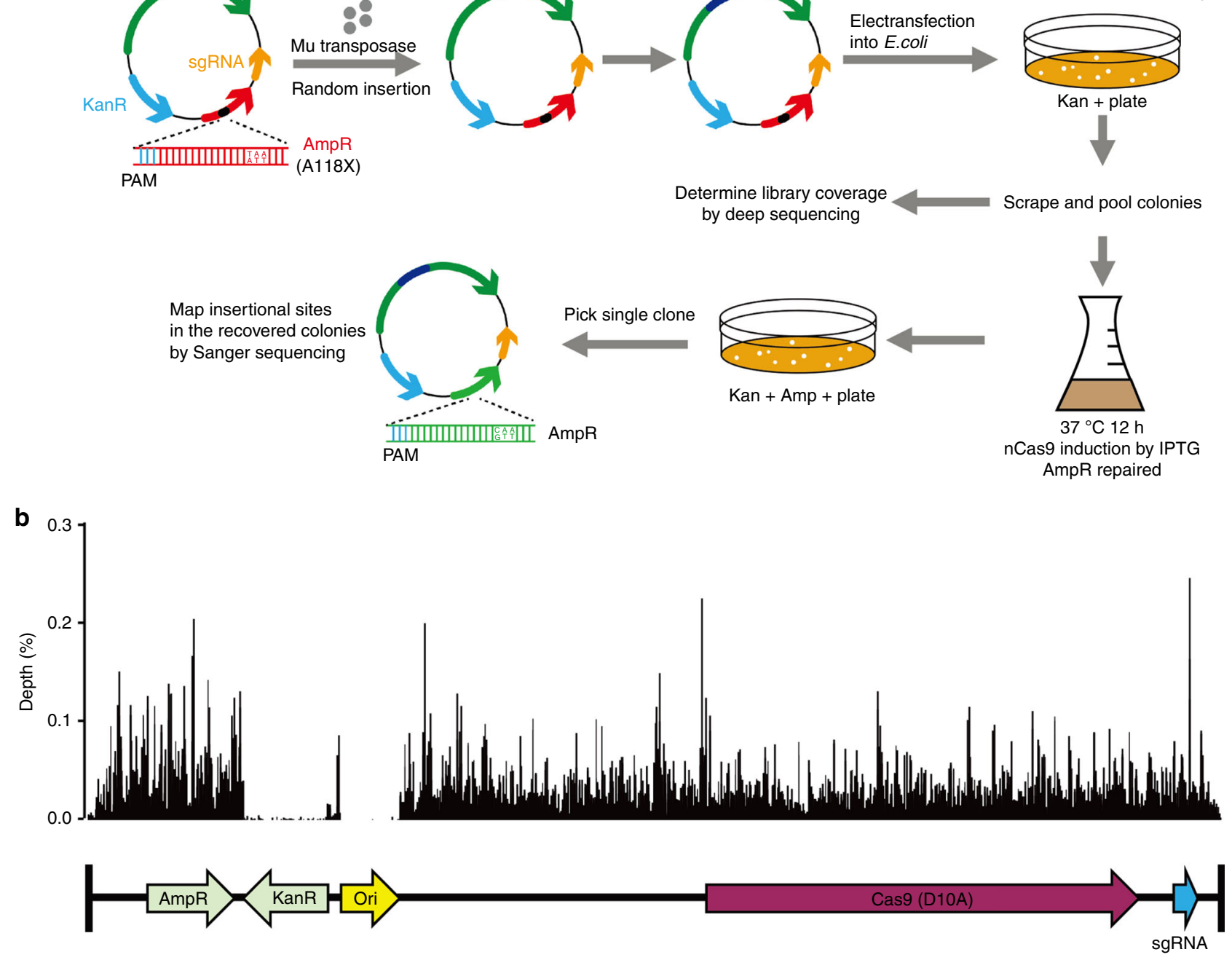

C

AmpR (A118X to X118Q)

GCGCGGTATTATCCCGTATTGACGCCGGGTAAGAGCAACTCGGTC

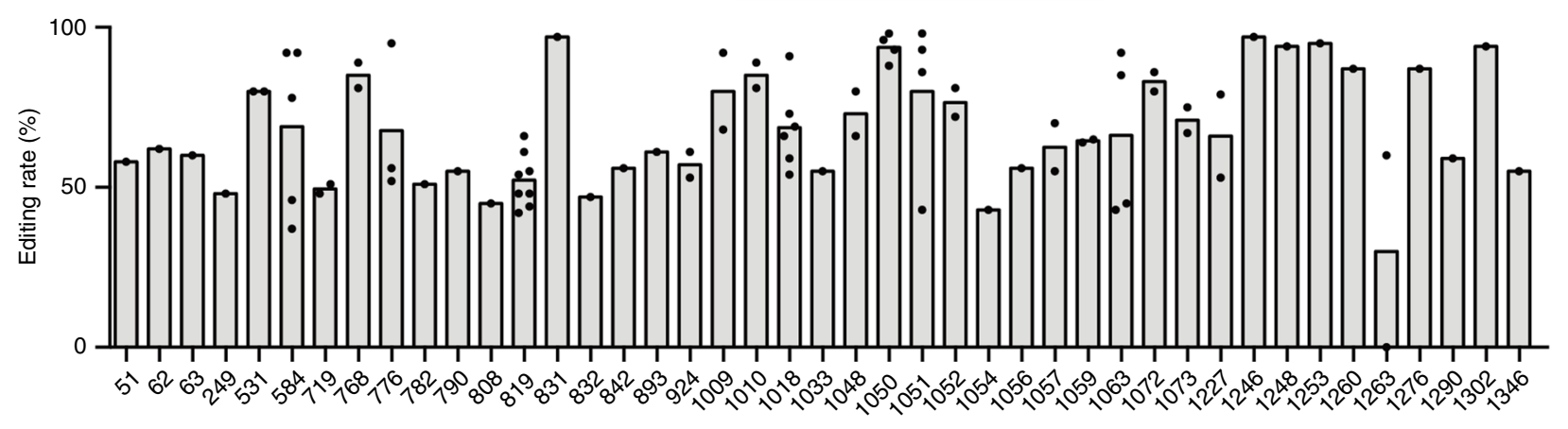

Insertion site (a.a.)

Fig. 1 Genetic screens for tolerant sites on nCas9 using transposon-mediated random mutagenesis. a Workflow. AmpR (A118X), ampicillin resistance gene bearing a premature stop codon. $\mathbf{b}$ Insertion site distribution pattern across the entire plasmid in the unscreened library, revealed by deep-seq. $\mathbf{c}$ nCas9 insertion sites in the screened library and the corresponding A > G conversion rates at the Amp resistance gene in the same plasmid, as revealed by Sanger sequencing. Each dot represents a single Amp-resistant colony, with a total of 84 colonies examined from three independent experiments. The AmpR sequence surrounding A118X is shown, with the targeted base and PAM highlighted in red and blue, respectively. A:T to G:C editing at the target would convert the stop codon to CAA (encoding Q). 
a

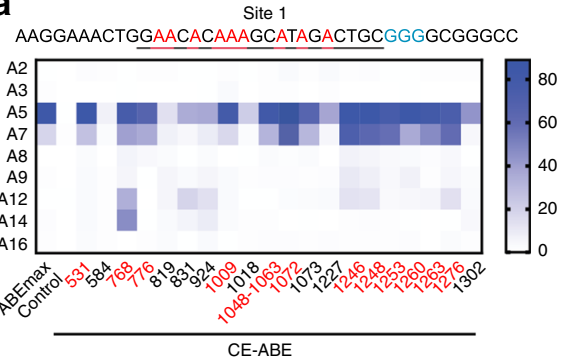

b

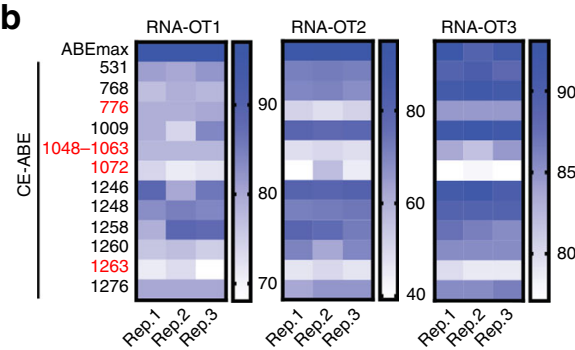

C

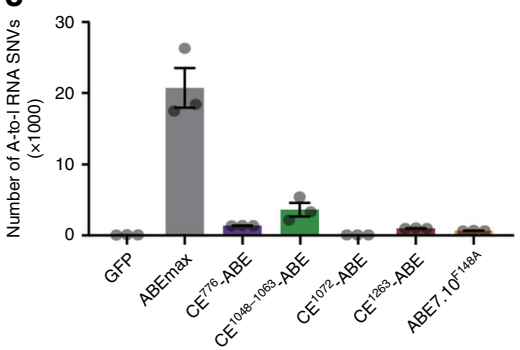

d

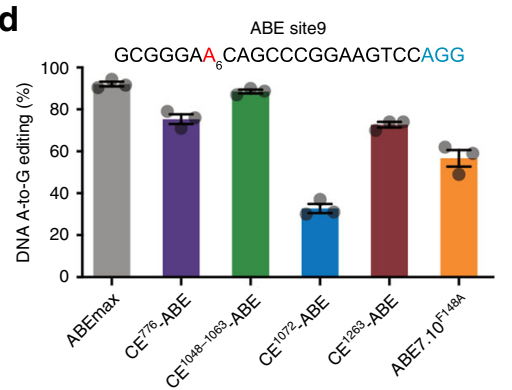

f
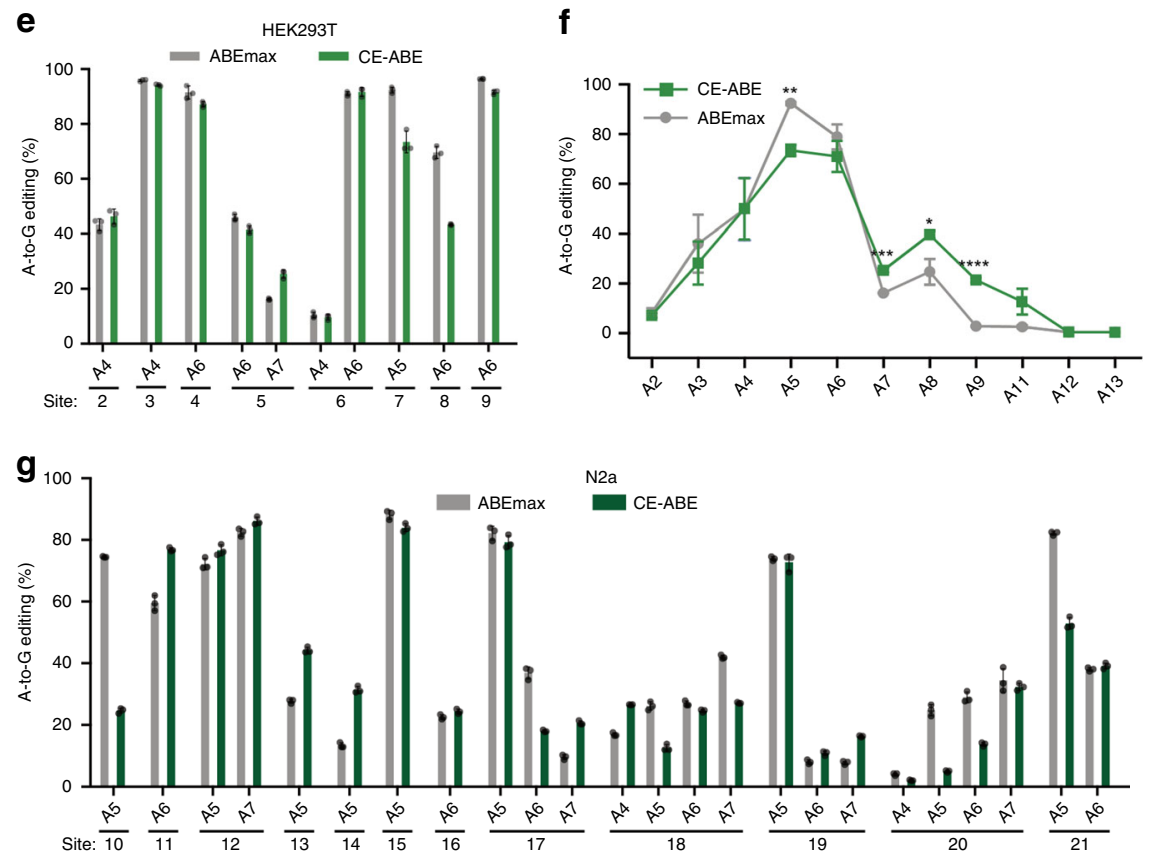

h
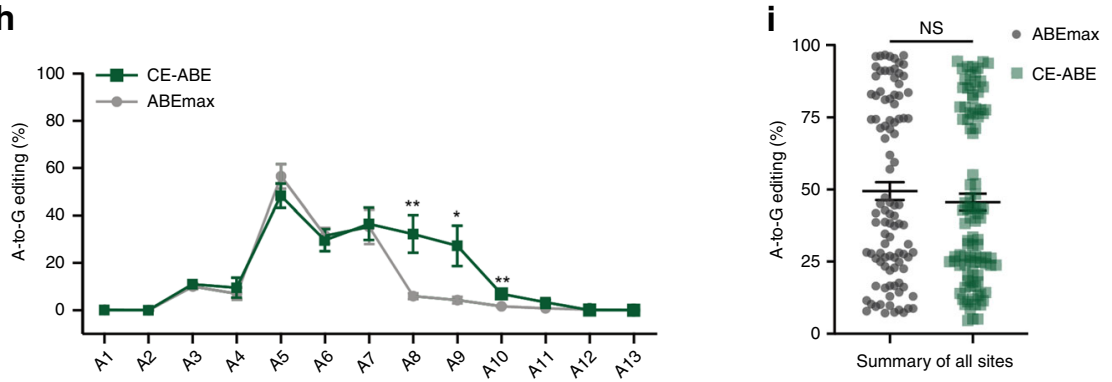

The CE strategy is applicable to CBEs bearing different Apobec proteins. Encouraged by the success in CE-ABEs, we sought to derive the $\mathrm{CE}$ versions of two distinct CBEs, the first being AncBE4max consisting of the APOBEC ancestor Anc689 linked to the $\mathrm{N}$ terminus of nCas9, which is highly active but presumably also highly non-specific ${ }^{17}$. To create the CE version of AncBE4max, we relocated Anc689 to position 1048-1063 in nCas9, replacing the native Cas9 sequence in the process; the resulting editor was termed $\mathrm{CE}^{1048-1063}$ - CBE. We also generated $\mathrm{CE}^{1071}$. $\mathrm{CBE}$. The second $\mathrm{CBE}$ we sought to optimize involves BE4-A3A, comprising APOBEC3A fused to the $\mathrm{N}$ terminus of nCas9. BE4$\mathrm{A} 3 \mathrm{~A}$ is one of the most active $\mathrm{CBEs}$ created so far, but also displays the highest guide RNA (gRNA)-independent off-target editing on $\mathrm{DNA}^{11,19}$. We have previously found that introducing 
Fig. 2 Characterization of CE-ABEs in mammalian (HEK293 and N2a) cells. a On-target editing in HEK293T cells by 20 CE-ABE variants at Site1, a known $A B E$ target ${ }^{24}$. Twelve CE-ABEs were as active as ABEmax (red). Data averaged from three biological replicates. $\mathbf{b}$ Off-target editing by the $12 \mathrm{CE}-\mathrm{ABEs}$ at three known RNA off-target sites of ABEmax we identified using RNA-seq in preliminary studies. Top four candidates with minimal off-target effects are highlighted (red). c, d On-target (c) and off-target (d) editing by the four candidates. e, f On-target editing by the optimal variant (CE1048-1063-ABE) benchmarked against ABEmax in HEK293T cells. Shown are the editing rates of the susceptible As at individual sites (e) and at all the As along the target site specified by the gRNA protospacers, as averaged from all the eight sites (f). $P=0.0014,0.0005,0.0199$, and 0.0001 for $A 5, A 7, A 8$, and $A 9$, respectively. $\mathbf{g}$, $\mathbf{h}$ Same as panels (e) and (f), except that the editors were compared in N2a cells. $P=0.0048,0.0127$ and 0.0021 for A8, A9, and A10, respectively. i Editing efficiencies on 20 sites, summarized from panels $(\mathbf{e})$ and $(\mathbf{g})$. NS not significant $(P=0.36)$. In a-h, the 20 test sites used (\#2-21) are described in Supplementary Table 1, and the values in the graphs are mean \pm s.e.m. $\left(n=3\right.$ independent biological replicates), with ${ }^{\star},{ }^{\star \star},{ }^{\star \star \star}$ and ${ }^{\star \star \star \star}$ representing $P<0.05,0.01,0.001$ and $<0.0001$, respectively (two-sided unpaired $t$ test).

Y130F into A3A in the context of BE3-A3A mitigates the offtarget effect only partially, with substantial off-target edits persisting in $\mathrm{BE} 3-\mathrm{A} 3 \mathrm{~A}(\mathrm{Y} 130 \mathrm{~F})$, which limits the use of the editor ${ }^{10}$. To minimize the off-target effect of $\mathrm{A} 3 \mathrm{~A}(\mathrm{Y} 130 \mathrm{~F})$, we replaced $\mathrm{rA} 1$ of $\mathrm{CE}^{1048-1063}-\mathrm{CBE}$ with $\mathrm{A} 3 \mathrm{~A}(\mathrm{Y} 130 \mathrm{~F})$, generating $\mathrm{CE}^{1048-1063}$ $\mathrm{A} 3 \mathrm{~A}(\mathrm{Y} 130 \mathrm{~F})$. We also generated $\mathrm{CE}^{1072}-\mathrm{A} 3 \mathrm{~A}(\mathrm{Y} 130 \mathrm{~F})$ and $\mathrm{BE} 4 \mathrm{max}-\mathrm{A} 3 \mathrm{~A}(\mathrm{Y} 130 \mathrm{~F})$ for comparison.

We first determined the effects of $\mathrm{CE}$ on on-target editing. To this end, we compared the on-target editing rates of $\mathrm{CE}^{1048-1063}$ $\mathrm{CBE}$ with that of AncBE4max at nine randomly selected target sites in HEK293T cells, finding that CE did not compromise the editing efficiency of AncBE4max (Fig. 3a, b) or alter its editing window (Fig. 3c). A similar result was seen when comparing CE ${ }^{1048-1063}$-A3A(Y130F) with BE4max-A3A(Y130F) (Fig. 3d-f). $\mathrm{A}$ unique advantage of $\mathrm{BE}-\mathrm{A} 3 \mathrm{~A}$ is that they can efficiently edit GC-rich and highly methylated regions, contrary to the traditional $\mathrm{CBEs}$ utilizing $\mathrm{rAl}^{19}$. To determine whether this important advantage of $\mathrm{A} 3 \mathrm{~A}$ is retained in $\mathrm{CE}^{1048-1063}-\mathrm{A} 3 \mathrm{~A}$ (Y130), we benchmarked $\mathrm{CE}^{1048-1063}-\mathrm{A} 3 \mathrm{~A}(\mathrm{Y} 130)$ against $\mathrm{YE} 1-$ $\mathrm{BE} 4 \mathrm{max}$, the most active $\mathrm{CBE}$ we and others reported ${ }^{10,12}$. We compared the two editors in HEK293T cells at three highly methylated target genes. Indeed, $\mathrm{CE}^{1048-1063}-\mathrm{A} 3 \mathrm{~A}(\mathrm{Y} 130 \mathrm{~F})$ clearly outperformed YE1-BE4max at all three sites (Fig. 3g, h), confirming that $\mathrm{CE}^{1048-1063}-\mathrm{A} 3 \mathrm{~A}(\mathrm{Y} 130 \mathrm{~F})$ was preferable over YE1-BE4max for highly efficient editing at methylated (and by inference, GC-rich) regions. Of note, at these sites, CE ${ }^{1048-1063}$ $\mathrm{A} 3 \mathrm{~A}(\mathrm{Y} 130 \mathrm{~F})$ was as active as $\mathrm{BE} 4 \mathrm{max}-\mathrm{A} 3 \mathrm{~A}(\mathrm{Y} 130 \mathrm{~F})$, but more active than BE3-A3A(Y130F), as expected (Fig. 3g, h).

Having demonstrated the CE did not alter the on-target editing by CBEs, we next determined whether CE could indeed selectively inhibit their off-target effects. In contrast to ABEs, which do not affect the genome, CBEs are known to display gRNAindependent off-target editing on the genome in addition to off-target effects on the transcriptome. We first evaluated the DNA off-target edits using GOTI (genome-wide off-target analysis by two-cell embryo injection), a highly sensitive and physiologically relevant assay for detecting random genomic offtarget edits. In this method, the editors were coexpressed with a sgRNA targeting the Tyrosinase gene, and the DNA from E12.5 embryos was sequenced at a depth of $\sim 30 \times$. AncBE4max created $\sim 773$ SNVs per embryo, $43 \times$ above the background in the GFP control (18 SNVs) (Fig. 4a, top; Supplementary Fig. 2a, b). In sharp contrast, only 19 and 31 SNVs per embryo were detected in the embryos treated with CE-CBE ${ }^{1048-1063}$ and the CE-CBE ${ }^{1072}$, respectively, each similar to the GFP control and to YE1-BE3, a highly specific BE3 variant ${ }^{10,12}$ (Fig. 4a, top; Supplementary Fig. 2a, b). As expected, in the SNVs detected in AncBE4maxtreated embryos, the SNV subsets resulting from C:G>T:A conversion predominated, constituting $92 \%$ of the total SNVs, whereas in the embryos treated with CE-CBEs, GFP, or YE1-BE3, the subsets were less abundant $(<68 \%$, but accurate quantification not feasible due to the scarcity of SNVs; Fig. 4a, bottom and Supplementary Fig. 2c). The same trend was observed for A3A- editors, with substantial numbers of SNVs (276) detected in BE3$\mathrm{A} 3 \mathrm{~A}(\mathrm{Y} 130 \mathrm{~F})$ but much less $(43-63)$ in $\mathrm{CE}^{1048-1063}-\mathrm{A} 3 \mathrm{~A}(\mathrm{Y} 130 \mathrm{~F})$ or $\mathrm{CE}^{1072}-\mathrm{A} 3 \mathrm{~A}(\mathrm{Y} 130 \mathrm{~F})$ (Fig. 4a and Supplementary Fig. 2). We conclude that CE markedly decreased DNA off-target effects of CBEs. Importantly, this decrease is not an artifact resulting from nonspecific inactivation of the CE-editors, as revealed by their robust editing at the on-target in the mouse embryos (Fig. 4b).

We next quantified off-target RNA editing using RNA-seq. BE4max created massive off-target edits $(\sim 3000)$ as expected, but interestingly, AncBE4max only 74 edits, presumably reflecting the property of Anc689 harnessed in the editor (Fig. 4c). Nevertheless, the off-target effect was clearly above the GFP control, and was completely eliminated in CE-CBE, which produced only 15 edits, a level indistinguishable from the background (Fig. 4c). For A3A editors, CE-mediated suppression of RNA off-target editing was much more pronounced, reducing the SNV numbers $100 \times$, from 2025 in $\mathrm{BE} 4 \mathrm{max}-\mathrm{A} 3 \mathrm{~A}(\mathrm{Y} 130 \mathrm{~F})$ to only 18 in $\mathrm{CE}^{1048-1063}-\mathrm{A} 3 \mathrm{~A}(\mathrm{Y} 130 \mathrm{~F})$, a level only slightly above the GFP background (11) (Fig. 4d).

These results show that the CE strategy is applicable to CBEs, enabling the creation of $\mathrm{CE}^{1048-1063}-\mathrm{A} 3 \mathrm{~A}(\mathrm{Y} 130 \mathrm{~F})$, a highly specific editor capable of robust editing at methylated or presumably GC-rich sequences.

\section{Discussion}

We have shown that CE is a powerful countermeasure of off-target effects of DNA base editors, which is much simpler than the conventional method of deaminase domain mutagenesis ${ }^{3,4,10-14}$. Although the off-target effects can also be countered by mutations, these mutations are identified on a case-by-case basis, typically via extensive structure-function analysis, contrary to CE, which requires no prior knowledge about the enzymes. We are aware that CE may be insufficient for fully eliminating off-target effects for some editors, but so may be mutations, and in this case, the two approaches may be used in conjunction to achieve the optimal effect, as illustrated in CE-A3A(Y130F), where CE and Y130F act together to minimize the off-targets on DNA. Indeed, CE is not mutually exclusive but complementary with other editor optimization methods, including the use of short-rigid linkers, an elegant strategy for narrowing the editing window ${ }^{13,14}$. Of note, we have recently found that for an RNA base editor comprising the ADAR2 deaminase domain fused to the programmable RNAbinding protein $\mathrm{dCasRx}$, inserting the deaminase domain into a flexible loop on the surface of dCasRx could reduce the off-target effects on RNA slightly $(1.8 \times)$ relative to a terminal fusion configuration $^{20}$. The data suggest that the CE method is perhaps generalizable to RNA base editors, assuming that the transposonbased screen on dCasRx can similarly yield optimal insertion sites analogous to the ones discovered in nCas9.

How exactly CE reduces the off-target effects is unclear. Offtarget editing of DNA base editors is caused by the deaminase moiety independent of nCas9, whereas the on-target editing is achieved when the deaminase moiety is brought into the proximity 

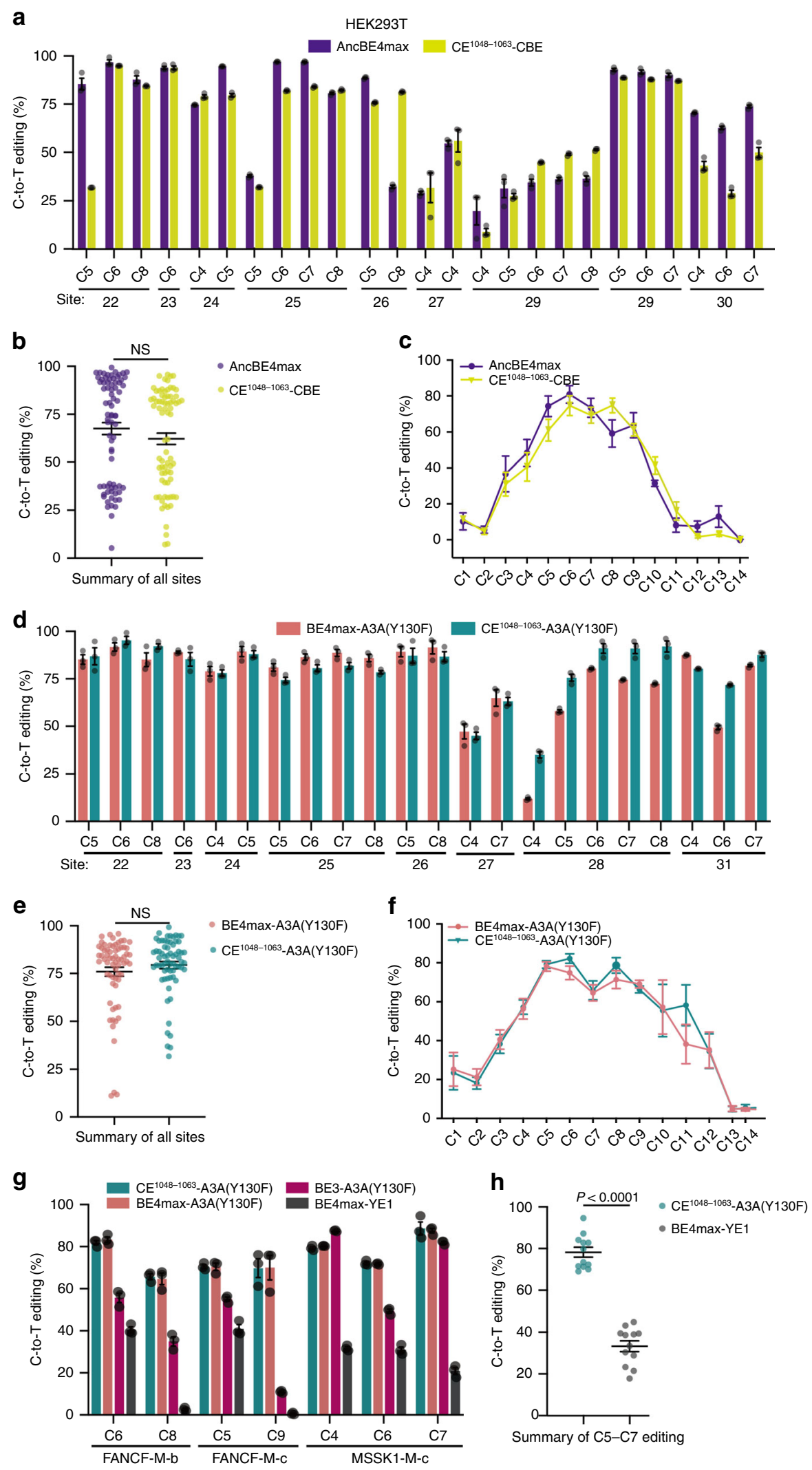

Fig. 3 On-target editing by CE ${ }^{1048-1063}-$ CBE and CE ${ }^{1048-1063}$-A3A(Y130F) in HEK293T cells. a-f $C^{1048-1063-C B E ~ w a s ~ b e n c h m a r k e d ~ a g a i n s t ~}$ AncBE4max (a-c), while CE1048-1063-A3A(Y130F) against BE4-A3A(Y130F) (d-f). The editing rates of the susceptible Cs are shown for individual sites (a, d) and as a pool (b, e). Also shown are the editing rates of all the $\mathrm{Cs}$ along the target region specified by the gRNA protospacers, as averaged from all the tested sites (c, f). The test sites used (\#22-31) are described in Supplementary Table 1. $\mathbf{g}, \mathbf{h}$ CE ${ }^{1048-1063}$-A3A editing at representative methylated sites ${ }^{14}$. The editing rates at susceptible Cs are displayed for individual sites ( $\mathbf{g}$ ) or as a pool (h). Values in the $\mathbf{a}, \mathbf{c}, \mathbf{d}, \mathbf{f}, \mathbf{g}$ are mean \pm s.e.m. from three biological replicates. NS not significant. 
a
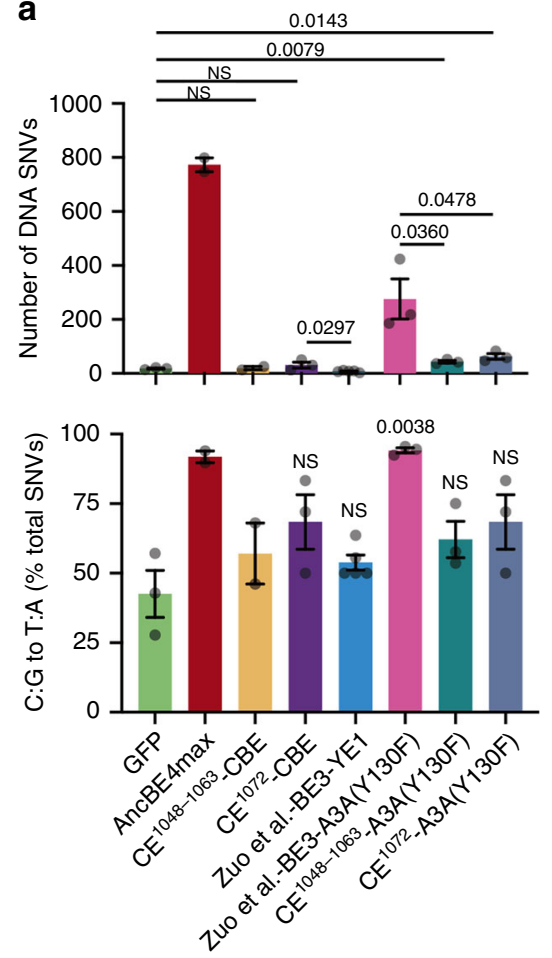

C

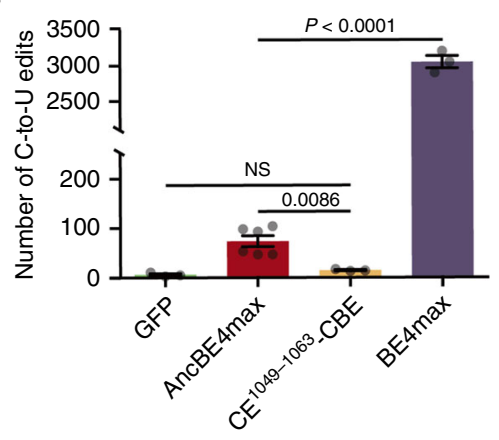

b

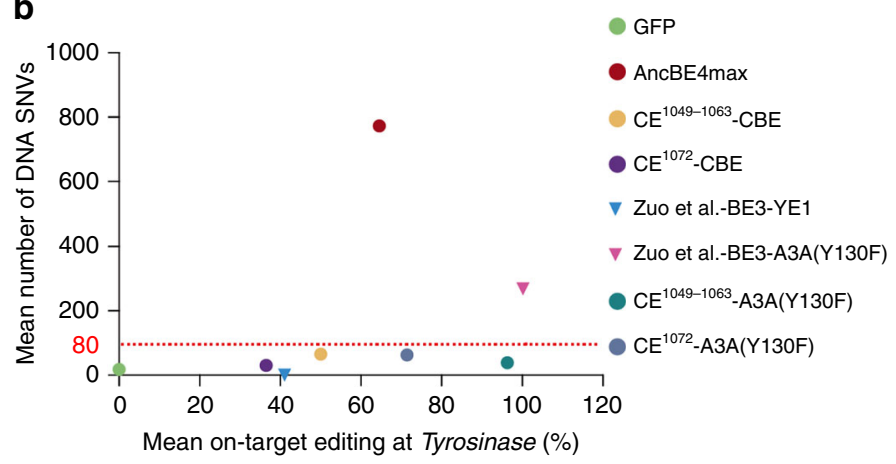

d

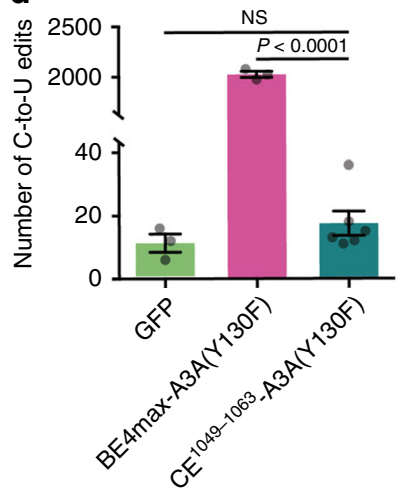

Fig. 4 Off-target effects of CE-CBEs. a, b, GOTI assay detecting off-target SNVs at the mouse genome. The bar graphs (a) display the total numbers of SNVs (top) and the proportions of the relevant subsets (bottom), while the scatter plot (b) displays the total SNV numbers and the on-target editing rates of the editors. The data were all generated in this study except for YE1-BE3 and BE3-A3A(Y130F), which were from refs. 5,10, respectively. Values in a, b are mean \pm s.e.m from two to three biological replicates as indicated by the dots. c, $\mathbf{d}$ RNA-seq analysis of off-target effects at the transcriptome for CE-CBE (c) and CE-A3A (d). Values in $\mathbf{c}, \mathbf{d}$ are mean \pm s.e.m for three or six biological replicates as indicated by the dots. NS not significant.

of the targets by nCas9. Embedding the deaminase domains in nCas9 might create some steric hindrance that hampers their free access to the off-targets, but not an on-target owing to their forced tethering via nCas9. This idea is testable by replacing the current XTEN linkers in the CE editors with longer, flexible linkers, which could relieve the putative steric hindrance. Besides, structural studies as exemplified by Doudna and colleagues ${ }^{21}$ should be valuable for providing conclusive evidence regarding the mechanisms of CE. Of note, circularly permutated nCas 9 has been created whose protein termini are placed at various positions ${ }^{22}$. It would be interesting to determine whether fusing the deaminase domains to the termini could also selectively compromise off-target editing.

We have used CE to successfully improve the specificity for both $\mathrm{ABE}$ and $\mathrm{CEB}$. In particular, $\mathrm{CE}^{1048-1063}-\mathrm{ABE}$ is as efficient as ABE7.10 ${ }^{\mathrm{F} 148 \mathrm{~A}}$ but slightly less specific, and so unable to outperform ABE7.10 ${ }^{\mathrm{F} 148 \mathrm{~A}}$. However, CE-ABE is far more specific than ABEmax, and our strategy for generating CE-ABE is very simple, contrary to the mutagenesis approach used to engineer ABE7.10 ${ }^{\mathrm{F} 148 \mathrm{~A}}$. In contrast to CE-ABE, CE ${ }^{1048-1063}-\mathrm{A} 3 \mathrm{~A}(\mathrm{Y} 130 \mathrm{~F})$ has unique advantages over the existing CBEs, being highly efficient at GC-rich and methylated sites while displaying little off-target editing on genomic DNA. As described above, this novel editor illustrates how CE and other strategies can be used together for base editor optimization.

Finally, we have designed a transposase-based platform and successfully used it to identify 43 sites in nCas9 tolerant of deaminase insertion. This information may be useful for optimizing other base editors such as the prime editor ${ }^{23}$. Interestingly, in a previous study, Doudna and colleagues delineated 175 hotspots on dCas9 tolerant of insertions of a PDZ domain ${ }^{16}$, but there is little overlap between these hot spots and the $43 \mathrm{CE}$ sites mapped in this study. This discrepancy might reflect a "sampling error" due to unsaturated screening. However, this scenario seems unlikely. We have performed a total of three screens, obtaining 
45,40 , and 37 colonies, respectively. Importantly, some insertion sites were recovered multiple times in almost every screen, suggesting that the screening has been saturating. We therefore favor the possibility that the discrepancy instead reflects the differences in the experimental design between the two studies, as Doudna and colleagues searched for dCas9 sites tolerant of PDZ insertion, and their screen readout is dCas9-mediated gene silencing. The two studies collectively suggest that although Cas9 tolerates insertions at numerous sites, the optimal sites may be proteinspecific. Nevertheless, the region in Cas9 between a.a. 1048 and 1063 identified in our study may be generally tolerant of diverse proteins, because this site is non-conserved and supportive of superior performance for both $\mathrm{ABE}$ and $\mathrm{CBE}$. This region could therefore serve as the starting point when applying the $\mathrm{CE}$ strategy to new proteins in the future.

\begin{abstract}
Methods
Plasmid construction. Primers and plasmids are listed in Supplementary Table 1. pCMV-nCas9-KanR-AmpR(A118X)-sgRNA, the all-in-one plasmid for insertion screening, was assembled from pCMV-ABEmax (Addgene 112095), pUC57-Kan (Addgene, 51132), and pGL3-U6-sgRNA (Addgene, 51133). The sgRNA expression vector for mammalian cells was constructed using BsaI-digested pGL3-U6sgRNA-EGFP with annealed DNA oligos (Supplementary Table 1). The sgRNA expression vector for GOTI was constructed by cloning annealed DNA oligos (Supplementary Table 1) into BbsI-digested pUC57-sgRNA (Addgene, 51132). CEABEs and CE-CBEs were derived from pCMV-ABEmax (Addgene 112095) and pCMV-AncBE4max (112094), respectively.
\end{abstract}

\section{Insertional library construction, characterization, and screening. TadA-TadA*} was PCR-amplified from pCMV-ABEmax (Addgene 112095) and cloned into a MuA-transposon vector (Supplementary Fig. 1a). The transposon was excised from the vector using $B s a \mathrm{I}$ digestion before random insertion into pCMV-nCas9-KanR$\operatorname{AmpR}(\mathrm{A} 118 \mathrm{X})$-sgRNA in an in vitro reaction containing $250 \mathrm{ng}$ transposon, $500 \mathrm{ng}$ nCas9 plasmid, and $1 \mu \mathrm{L}$ of MuA transposase (F-701, Thermo Fisher). The reaction was incubated at $30^{\circ} \mathrm{C}$ for $1 \mathrm{~h}$ to achieve random insertion, followed by $75^{\circ} \mathrm{C}$ for $10 \mathrm{~min}$ to inactivate the MuA transposase. The DNA was precipitated, resuspended in $5 \mu \mathrm{L}$ deionized water, and electroporated into $100 \mu \mathrm{L}$ BL21(DE3)electrocompetent cells (Shanghai Weidi Biotechnology, EE1002). A total of $1 \mathrm{~mL}$ SOC media were then added and the bacteria were cultured at $37^{\circ} \mathrm{C}$ for $1 \mathrm{~h}$. The cells were then plated out on several LB agar plates containing $10 \mu \mathrm{g} / \mathrm{mL}$ kanamycin and incubated at $37^{\circ} \mathrm{C}$ overnight. The colonies were then collected by scraping, characterized, and screened. Specifically, to characterize the unscreened library, the insertional sites were deep-sequenced using an Illumina HiSeq X Ten $(2 \times 150 \mathrm{PE})$ at the Novogene Bioinformatics Institute (Beijing, China). All cleaned reads were first mapped to the backbone sequence using BWA v0.7.16 with the default parameters. The soft clipped reads were extracted and then mapped to the insertion sequence. All mapped soft clipped reads were checked and the breakpoints were recorded as insertion sites. To screen the library, the scraped cells were resuspended in $100 \mathrm{~mL} \mathrm{LB}$ containing $500 \mu \mathrm{M}$ IPTG. The culture was incubated for $12 \mathrm{~h}$ to induce nCas9 expression and repair the AmpR (A118X) mutation. Decreasing amounts of the culture $(5 \mathrm{~mL}, 1 \mathrm{~mL}, 500 \mu \mathrm{L}, 100 \mu \mathrm{L})$ were then plated out on $15-\mathrm{cm} \mathrm{LB}$ agar plates supplemented with ampicillin $(10 \mu \mathrm{g} / \mathrm{mL})$ and kanamycin $(10 \mu \mathrm{g} / \mathrm{mL})$. After an overnight incubation, the colonies were picked and subjected to Sanger sequencing to evaluate base editing at AmpR (A118X) and to determine the TadA-TadA* insertion sites.

Cell culture and transfection. HEK293T (ATCC CRL-3216) and Neuro-2a (N2a) (ATCC HTB-96) cells were cultured in Dulbecco's modified Eagle's medium (DMEM) (Hyclone, SH30243.01) supplemented with 10\% fetal bovine serum (v/v) (Gemini, 900-108) and penicillin and streptomycin (Gibco, 15140122). Cells were passaged once every 3 days and incubated at $37^{\circ} \mathrm{C}$ with $5 \% \mathrm{CO}_{2}$. All cells used in the experiment have been tested to exclude mycoplasma contamination. To evaluate the CE-ABEs, HEK293T cells were seeded on poly-D-lysine- (Sigma, P4707) coated 12-well plates (JET-BIOFIL, TCP010012) and transfected about $14 \mathrm{~h}$ at $\sim 80 \%$ density as per the manufacturer's protocols (Thermo Fisher Scientific, 11668019). Editor-expressing vectors (700 ng) were co-transfected with corresponding sgRNA-GFP plasmids (300 ng), and the cells with the highest $5 \%$ of GFP signal isolated by fluorescence-activated cell sorting $48 \mathrm{~h}$ later. However, for the experiment in Fig. 2a, b, "all-in-one" plasmids $(1 \mu \mathrm{g})$ expressing CE-ABE-P2A-GFP together with gRNA targeting Site1 was transfected instead. The isolated cells were analyzed for DNA and RNA editing as described below.

On-target genome editing in HEK293T and N2a cells. Genomic DNA was extracted using QuickExtract ${ }^{\mathrm{TM}}$ DNA Extraction Solution (Lucigen). The fragments encompassing the target sites ( $200 \mathrm{bp}$ ) were PCR-amplified using Phanta Max Super-Fidelity DNA polymerase (Vazyme, P505-03); the primers used are listed in Supplementary Table 1 . The amplicons were analyzed by deep sequencing on the Illumina Hiseq X Ten $(2 \times 150 \mathrm{PE})$ platform. The adapter pair of the paired-end reads were removed using AdapterRemoval version 2.2.2, and paired-end read alignments of $11 \mathrm{bp}$ or more bases were combined into a single consensus read. All the processed reads were then mapped to the target sequences using the BWA-MEM algorithm (BWA v0.7.16). For each site, the mutation rate was calculated using bam-read count with parameters $-q 20-b 30$. Indels were calculated based on the reads containing at least one inserted or deleted nucleotide in the protospacer. Indel frequency was expressed as the number of indelcontaining reads/total mapped reads.

RNA off-target editing in HEK293T cells. Cells were lysed in TRIzol reagent (Invitrogen, 15596026) and total RNA was extracted. For evaluation of off-target editing at specific sites, mRNA was reverse transcribed using HiScript II Q RT SuperMix Kit (Vazyme, R223-01) and the predicted off-targets were amplified using Phanta Max Super-Fidelity DNA polymerase (Vazyme, P505-03) with the primers listed in Supplementary Table 1. The amplicons were analyzed by Sanger sequencing and the editing rates were calculated using the following tool: https://moriaritylab. shinyapps.io/editr_v10/. For profiling of global RNA off-target editing, RNA samples were sequenced using an Illumina HiSeq X Ten $(2 \times 150 \mathrm{PE})$ at the Novogene Bioinformatics Institute (Beijing, China), at a depth of $\sim 20$ million reads per sample. The reads were mapped to the human reference genome (hg38) by STAR software (Version 2.5.1); annotation from GENCODE version v30 was used. After removing duplication, variants were identified using GATK HaplotypeCaller (version 4.1.2) and filtered with quality by depth) $<2$. All variants were verified and quantified by bamreadcount with parameters $-q 20-b 30$. The depth for a given edit had to be at least $10 \times$ and these edits were required to have at least $99 \%$ of reads supporting the reference allele in the wild-type samples. Finally, only A-to-G (for ABEs) or C-to-T (for CBEs) edits in the transcribed strand were considered for downstream analysis

GOTI assay. gRNA targeting the Tyrosinase gene and the mRNA encoding the editors were prepared as described ${ }^{4}$. Briefly, the gRNA was transcribed in vitro using the TranscriptAid T7 High Yield Transcription Kit (Thermo Fisher Scientific) with the primer listed in the Supplementary Table 1. sgRNAs were purified using the MEGAclear Kit (Thermo Fisher Scientific). The editor mRNAs were transcribed using mMESSAGE mMACHINE T7 Ultra Kit (Ambion, Life Technologies, AM1345) and purified using RNeasy Protect Mini Kit (Qiagen, 74124). All the subsequent steps were also performed as previously described ${ }^{4}$, but with one important modification in that the edited and non-edited blastomeres are now independently grown in distinct foster mothers to help ensure the healthy development of the edited embryos. Specifically, we obtained two-cell embryos on the B6D2F1(C57BL/6 $\times$ DBA2J) background, transplanted each blastomere to a recipient zona pellucida, injected the RNA mixture into one of the blastomeres, and transplant both the injected and control blastomeres into a respective foster ICR (albino) mother together with 6-8 ICR (albino) embryo. The embryos were analyzed at E12.5 by targeted sequencing for on-target editing and by whole-genome sequencing (WGS) for gRNA-independent off-target editing as usual. Briefly, WGS was performed at mean coverage of $30 \times$ using an Illumina HiSeq X Ten. Raw reads were trimmed with Trimmomatic (v0.36) and duplicates were removed using Sambamba (v0.6.7) before mapping qualified reads to the mouse reference genome (mm10) using BWA (v0.7.16). Three algorithms, Mutect2 (v3.5), Lofreq (v2.1.2), and Strelka (v2.7.1), were used to identify de novo variants, with the paired noninjected sample in the same embryo serving as a control. The subset of the SNVs reported by all three algorithms was considered the true variants. The gRNAdependent potential off-target sites bearing the NRG PAM were predicted using CasOT (http://casot.cbi.pku.edu.cn/) and Cas-OFFinder (http://www.rgenome.net/ cas-offinder/). The filter setting for CasOT was $\leq 2$ and $\leq 3$ mismatches for the seed and non-seed regions, respectively, whereas the setting for Cas-OFFinder was $\leq 3$ mismatches for the 20 -nucleotide protospacer.

Statistical analysis. No statistical methods were used to predetermine sample sizes. All values are shown as mean \pm s.e.m. Unpaired Student's $t$ test (two-tailed) was used for comparisons and $p<0.05$ was considered to be statistically significant.

Reporting summary. Further information on research design is available in the Nature Research Reporting Summary linked to this article.

\section{Data availability}

High-throughput sequencing data are available in the NCBI Sequence Read Archive database (https://www.ncbi.nlm.nih.gov/bioproject/?term=PRJNA660112). Source data are provided with this paper.

Received: 20 July 2020; Accepted: 19 October 2020; Published online: 27 November 2020 


\section{References}

1. Rees, H. A. \& Liu, D. R. Base editing: precision chemistry on the genome and transcriptome of living cells. Nat. Rev. Genet. 19, 770-788 (2018).

2. Anzalone, A. V., Koblan, L. W. \& Liu, D. R. Genome editing with CRISPR-Cas nucleases, base editors, transposases and prime editors. Nat. Biotechnol. 38, 824-844 (2020).

3. Grünewald, J. et al. Transcriptome-wide off-target RNA editing induced by CRISPR-guided DNA base editors. Nature 569, 433-437 (2019).

4. Zhou, C. et al. Off-target RNA mutation induced by DNA base editing and its elimination by mutagenesis. Nature https://doi.org/10.1038/s41586-019-13140 (2019).

5. Zuo, E. et al. Cytosine base editor generates substantial off-target singlenucleotide variants in mouse embryos. Science 364, 289-292 (2019).

6. Jin, S. et al. Cytosine, but not adenine, base editors induce genome-wide offtarget mutations in rice. Science 364, 292-295 (2019).

7. Gaudelli, N. M. et al. Programmable base editing of $A \cdot T$ to $\mathrm{G} \bullet \mathrm{C}$ in genomic DNA without DNA cleavage. Nature 551, 464-471 (2017).

8. Gapinske, M. et al. CRISPR-SKIP: programmable gene splicing with single base editors. Genome Biol. 19, 1-11 (2018).

9. Mao, S., Liu, Y., Huang, S., Huang, X. \& Chi, T. Site-directed RNA editing (SDRE): off-target effects and their countermeasures. J. Genet. Genomics 46, 531-535 (2019).

10. Zuo, E. et al. A rationally engineered cytosine base editor retains high ontarget activity while reducing both DNA and RNA off-target effects. Nat. Methods 17, 600-604 (2020).

11. Doman, J. L., Raguram, A., Newby, G. A. \& Liu, D. R. Evaluation and minimization of Cas9-independent off-target DNA editing by cytosine base editors. Nat. Biotechnol. 38, 620-628 (2020).

12. Kim, Y. B. et al. Increasing the genome-targeting scope and precision of base editing with engineered Cas9-cytidine deaminase fusions. Nat. Biotechnol. 35 , 371-376 (2017).

13. Tan, J., Zhang, F., Karcher, D. \& Bock, R. Engineering of high-precision base editors for site-specific single nucleotide replacement. Nat. Commun. 10, 439 (2019).

14. Tan, J., Zhang, F., Karcher, D. \& Bock, R. Expanding the genome-targeting scope and the site selectivity of high-precision base editors. Nat. Commun. 11, 629 (2020).

15. Haapa, S., Taira, S., Heikkinen, E. \& Savilahti, H. An efficient and accurate integration of mini-Mu transposons in vitro: a general methodology for functional genetic analysis and molecular biology applications. Nucleic Acids Res. 27, 2777-2784 (1999).

16. Oakes, B. L. et al. Profiling of engineering hotspots identifies an allosteric CRISPR-Cas9 switch. Nat. Biotechnol. 34, 646-651 (2016).

17. Koblan, L. W. et al. Improving cytidine and adenine base editors by expression optimization and ancestral reconstruction. Nat. Biotechnol. 36, 843-848 (2018).

18. Nishimasu, H. et al. Crystal structure of Cas9 in complex with guide RNA and target DNA. Cell 156, 935-949 (2014).

19. Wang, X. et al. Efficient base editing in methylated regions with a human apobec3a-cas9 fusion. Nat. Biotechnol. 36, 946 (2018).

20. Liu, Y., Mao, S. \& Chi, T. REPAIRx, a specific yet highly efficient programmable A > I RNA base editor. EMBO J. https://doi.org/10.15252/ embj.2020104748 (2020).

21. Lapinaite, A. et al. DNA capture by a CRISPR-Cas9-guided adenine base editor. Science 369, 566-571 (2020).

22. Oakes, B. L. et al. CRISPR-Cas9 circular permutants as programmable scaffolds for genome modification. Cell 176, 254-267.e16 (2019).
23. Anzalone, A. V. et al. Search-and-replace genome editing without doublestrand breaks or donor DNA. Nature 576, 149-157 (2019).

24. Grünewald, J. et al. CRISPR DNA base editors with reduced RNA off-target and self-editing activities. Nat. Biotechnol. 37, 1041-1048 (2019).

\section{Acknowledgements}

We thank Haibo Zhou for discussion and Yidi Sun for help with bioinformatics. We also thank the Molecular and Cell Biology Core Facility (MCBCF) at the School of Life Science and Technology, ShanghaiTech University for providing technical support. This work is supported by National Key R\&D Program (2016YFA0500903) and National Science Foundation of China (81830004).

\section{Author contributions}

Y.L., C.Z., and S.H. conceived of the project under the guidance of H.Y., X.H., and T.C. Y.L., C.Z., and S.H. performed the genetic screen. Y.L., L.D., and W.T. characterized the editors in vitro with help from S.M. and Y.Z., Y.W., C.Z., and Y.L. performed the GOTI experiments. S.H. and J.H. performed bioinformatics analysis with help from Y.S.Z., Y.L., and T.C. analyzed the data and wrote the manuscript with input from S.H. and C.Z.

\section{Competing interests}

The authors declare no competing interests.

\section{Additional information}

Supplementary information is available for this paper at https://doi.org/10.1038/s41467020-19690-0.

Correspondence and requests for materials should be addressed to H.Y., X.H. or T.C.

Peer review information Nature Communications thanks Sangsu Bae and the other, anonymous, reviewer(s) for their contribution to the peer review of this work. Peer reviewer reports are available.

Reprints and permission information is available at http://www.nature.com/reprints

Publisher's note Springer Nature remains neutral with regard to jurisdictional claims in published maps and institutional affiliations.

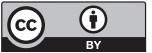

Open Access This article is licensed under a Creative Commons Attribution 4.0 International License, which permits use, sharing, adaptation, distribution and reproduction in any medium or format, as long as you give appropriate credit to the original author(s) and the source, provide a link to the Creative Commons license, and indicate if changes were made. The images or other third party material in this article are included in the article's Creative Commons license, unless indicated otherwise in a credit line to the material. If material is not included in the article's Creative Commons license and your intended use is not permitted by statutory regulation or exceeds the permitted use, you will need to obtain permission directly from the copyright holder. To view a copy of this license, visit http://creativecommons.org/ licenses/by/4.0/.

(C) The Author(s) 2020 